\title{
A Universal Relationship between Optimum Drift Voltage and Resolving Power
}

\author{
Ansgar T. Kirk, Kai Bakes, Stefan Zimmermann
}

Leibniz Universität Hannover, Institute of Electrical Engineering and Measurement Technology, Department of Sensors and Measurement Technology, Appelstr. 9A, 30167 Hannover, Germany

\begin{abstract}
The drift voltage is one of the key experimental parameters of any drift tube ion mobility spectrometer. In this work, we show that a universal relationship between optimum drift voltage and the resolving power reached at this point exists, governed only by temperature and ion charge state. With these two quantities known, the measured optimum drift voltage and resolving power combination can be used to estimate the ideality of the drift conditions inside a drift tube, since any deviation from the theoretical values must be caused by non-idealities in the ions' drift. Analyzing drift voltage sweeps from nine different ion mobility spectrometers, a continuous increase in drift tube ideality over the past is observed, reaching from less than $50 \%$ thirty years ago to $99 \%$ for a current design based on printed circuit boards. Furthermore, possible causes for the observed non-idealities are discussed.
\end{abstract}

Keywords: Analytical model, drift tube, ideality, non-idealities, ion mobility spectrometry

\section{Introduction}

Drift tube ion mobility spectrometers characterize ions based on their motion through a neutral drift gas under the influence of an electrical field. Unsurprisingly, the strength of this field or respectively the total voltage applied across the drift tube is one of the key experimental parameters. Thus, analytical models to understand its influence on both the resolving power [1-4] and the limits of detection [4; 5] have been developed. The resolving power, defined as the ratio of drift time to peak width at half height, of a drift tube ion mobility spectrometer with a finite speed of shutter and amplifier can be described using eq. 1.

$$
R_{p}=\frac{t_{D}}{w_{0,5}}=\frac{\frac{L^{2}}{K U_{D}}}{\sqrt{w_{\text {min }^{2}}+\frac{16 \mathrm{k}_{\mathrm{B}} T \ln 2}{Z \mathrm{e} U_{D}}\left(\frac{L^{2}}{K U_{D}}\right)^{2}}}=\frac{1}{\sqrt{\left(\frac{w_{\min } K U_{D}}{L^{2}}\right)^{2}+\frac{16 \mathrm{k}_{\mathrm{B}} T \ln 2}{Z \mathrm{e} U_{D}}}}
$$

Its parameters are the length of the drift tube $L$, the drift voltage $U_{d}$ applied across it, the minimum peak width $w_{\min }$ achievable due to the initial ion packet width and amplifier distortion, the ions' mobility $K$ and their charge state $z$ as well as the absolute temperature $T$.

It can be interpreted as a geometric average between the inverse of two partial resolving powers that are represented by the two terms in the denominator of the right hand side of eq. 1 . In the limit of very high drift voltages, the second term describing the diffusion vanishes and only the first term remains. This can be called the minimum-width-limited resolving power, given by eq. 2 .

$$
R_{p, w_{\text {min }}}=\frac{L^{2}}{w_{\text {min }} K U_{D}}
$$

In the limit of very low drift voltages, the first term vanishes and only the contribution of the diffusion remains, leading to the diffusion-limited resolving power described by eq. 3. The diffusion limited resolving power is often used in estimations instead of eq. 1 due to its simplicity. However, very few ion mobility spectrometers will actually be operated in the completely diffusion-limited region due to massive loss of sensitivity [5]. Thus, analysis by only the diffusion-limited resolving power will often be erroneous. 


$$
R_{p, \text { diff }}=\sqrt{\frac{z \text { e } U_{D}}{16 \mathrm{k}_{\mathrm{B}} T \ln 2}}
$$

Therefore, a deeper understanding of the complex behavior of eq. 1 is of interest to gain a better understanding of drift tube resolving power. While the sensitivity increases continuously with higher drift voltages [5], the resolving power must possess an optimum as one of its limitations increases and the other decreases with increasing drift voltage. The optimum drift voltage $\mathrm{U}_{\text {opt }}$ is given by eq. 4, which can be found from the derivative of eq. 1 [4]. At this point, a balance between the limitations through diffusion and minimum width is obtained. Interestingly, at this point the contribution of diffusion to the peak width is exactly twice the contribution of the minimum width [4], which is helpful to know for many estimations.

$$
U_{\text {opt }}=\sqrt[3]{\frac{8 \mathrm{k}_{\mathrm{B}} T \ln 2}{z \mathrm{e} K^{2}} \frac{L^{4}}{w_{\min ^{2}}}}
$$

Inserting eq. 4 into eq. 1 yields eq. 5, describing the optimum resolving power $R_{\text {opt }}$ obtained at $U_{\text {opt }}$.

$$
R_{\text {opt }}=\sqrt[3]{\frac{z \mathrm{e}}{24 \sqrt{3} \ln 2 \mathrm{k}_{\mathrm{B}} T K} \frac{L^{2}}{w_{\min }}}
$$

All these relationships are visualized in Figure 1, which shows the diffusion-limited resolving power, the minimum-width-limited resolving power, the resulting resolving power and its optimum. As operation at any drift voltage can be described for any drift tube ion mobility spectrometer based on its operation at its optimum drift voltage [5], this is the most important operating point to analyze.

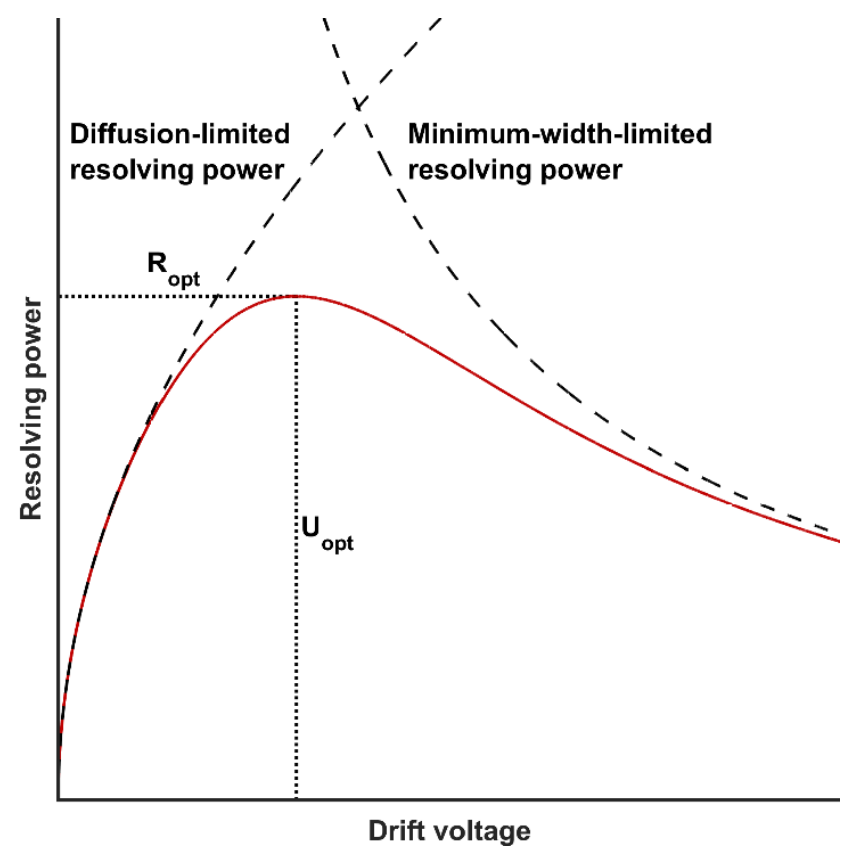

Figure 1: Diffusion-limited resolving power (left dashed line), minimum-width-limited resolving power (right dashed line), the resulting resolving power (solid red line) and its optimum (dotted lines).

However, while the equations for the optimum drift voltage and the optimum resolving power are simpler than the full resolving power equation eq. 1, they still depend on a multitude of variables, complicating their evaluation in most real-world cases. Thus, further analysis is of interest. By solving eq. 4 for e.g. the drift length $L$ and inserting the result into eq. 5, a universal relationship between the optimum drift voltage and the optimum resolving power can be obtained [6], which can only be influenced by changing either the drift tube temperature 
or the ion charge state. This was interpreted as a universal limitation for the resolving power of drift tube ion mobility spectrometers. However, it can also be arranged into the same form as eq. 3, yielding the relationship given by eq. 6 .

$$
R_{o p t}=\sqrt{\frac{z \mathrm{e} U_{o p t}}{24 \mathrm{k}_{\mathrm{B}} T \ln 2}}
$$

The resolving power of an ion mobility spectrometer at its optimum drift voltage is thus always $\sqrt{3 / 2}$ lower than the diffusion-limited resolving power for this drift voltage. This holds true for any drift tube length, shutter opening time, amplifier speed, pressure, drift gas and ion mobility. To illustrate this relationship further, the theoretical resolving power curves for arbitrary IMS operated at the same temperature are shown in Figure 2. They all follow the diffusion-limited resolving power shown as the dotted red line until deviating due to the minimum-width-limited resolving power. All of their maxima fall onto the curve described by eq. 6, which is shown as the solid blue line.

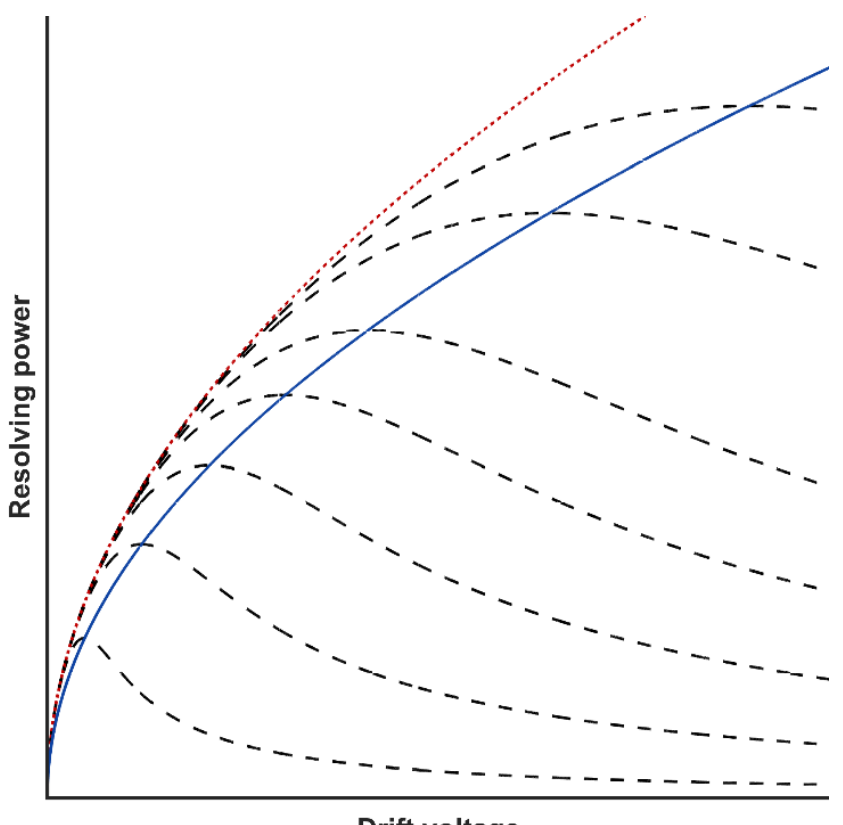

Drift voltage

Figure 2: Theoretical resolving power curves for different ion mobility spectrometers (dashed black lines), the diffusionlimited resolving power (dotted red line) and the optimum resolving power curve (solid blue line).

Eq. 6 is preferable to other methods of theoretical resolving power analysis in most cases. Compared to eq. 1, it is much simpler - no knowledge about the IMS is required to test its performance apart from the drift tube temperature and the ion charge state. Compared to eq. 3, it is significantly closer to the real application, as an ion mobility spectrometer should be operated at or above its optimum drift voltage to maintain a high signal-to-noise ratio [5]. Furthermore, the charge state can be assumed to be one for every ion source but electrospray ionization (ESI) and in order to achieve a resolving power accuracy of $\pm 1 \%$ at room temperature, the temperature needs to be known only within $\pm 6 \mathrm{~K}$, which is easily measurable or even guessable. Assuming a charge state of one, eq. 6 simplifies to eq. 7 for use in the analysis.

$$
R_{o p t}=26.4 \sqrt{\frac{U_{o p t}}{T}}
$$

\section{Experimental data}

Drift voltage sweeps are a well-known tool for IMS characterization and modelling and thus data from several older modelling papers is readily available. The data used in the analysis was taken from publications of Rokushika et. al. [7], Watts et. al. [8], Siems et. al. [2], Kanu et al. [3], Kirk et. al. [4; 5] and Bohnhorst et. al. [9]. 
In total, 44 drift voltage sweeps from nine different drift tube ion mobility spectrometers at eight different temperatures and many different minimum widths were analyzed. The main features of these drift tube designs are summarized in Table 1. Instruments sharing the same design but utilizing a different length were analyzed together.

Table 1: Overview of the different drift tubes analyzed in this study. Values are rounded for better readability. Question marks indicate values not given in the cited publications.

\begin{tabular}{|c|c|c|c|c|c|c|c|}
\hline Publication & $\begin{array}{c}\text { Length } \\
\text { in } \mathrm{mm}\end{array}$ & $\begin{array}{c}\text { Diameter } \\
\text { in } \mathrm{mm}\end{array}$ & $\begin{array}{c}\text { Voltage } \\
\text { in } \mathrm{kV}\end{array}$ & $\begin{array}{c}\text { Temperature } \\
\text { in }{ }^{\circ} \mathrm{C}\end{array}$ & $\begin{array}{c}\text { Pressure } \\
\text { in bar }\end{array}$ & Drift gas & Ion source \\
\hline Rokushika et. al. [7] & 80 & 43 & $0.3-3$ & 190 & 1 & $\mathrm{~N}_{2}$ & ${ }^{63} \mathrm{Ni}$ \\
\hline Watts et. al. [8] & $80-440$ & $?$ & $0.5-22$ & 35 & $?$ & $?$ & $?$ \\
\hline Siems et. al. [2] & 116 & 49 & $1.9-4.4$ & $175-325$ & 0.9 & $?$ & ${ }^{63} \mathrm{Ni}$ \\
\hline Kanu et. al. [3] & 68 & 22 & $0.8-5$ & 25 & 0.9 & Air & ${ }^{63} \mathrm{Ni}$ \\
\hline Kirk et. al. [4; 5] & $75-153$ & $15-21$ & $1-25$ & 25 & 1 & Air & ${ }^{3} \mathrm{H}$ \\
\hline Bohnhorst et. al. [9] & 50 & 15 & $1.3-3.7$ & 25 & 1 & Air & ${ }^{3} \mathrm{H}$ \\
\hline
\end{tabular}

\section{Results and discussion}

The optimum resolving power plotted across the optimum drift voltage divided by the drift tube's temperature from all these sweeps is shown in Figure 3. Results from the same drift tube system are plotted using the same symbol. Furthermore, the result from eq. 7 is plotted as the solid red line. It should be noted that due to the logarithmic scale, the square root turns into a line with slope 0.5 and any multiplicative error turns into an offset. 


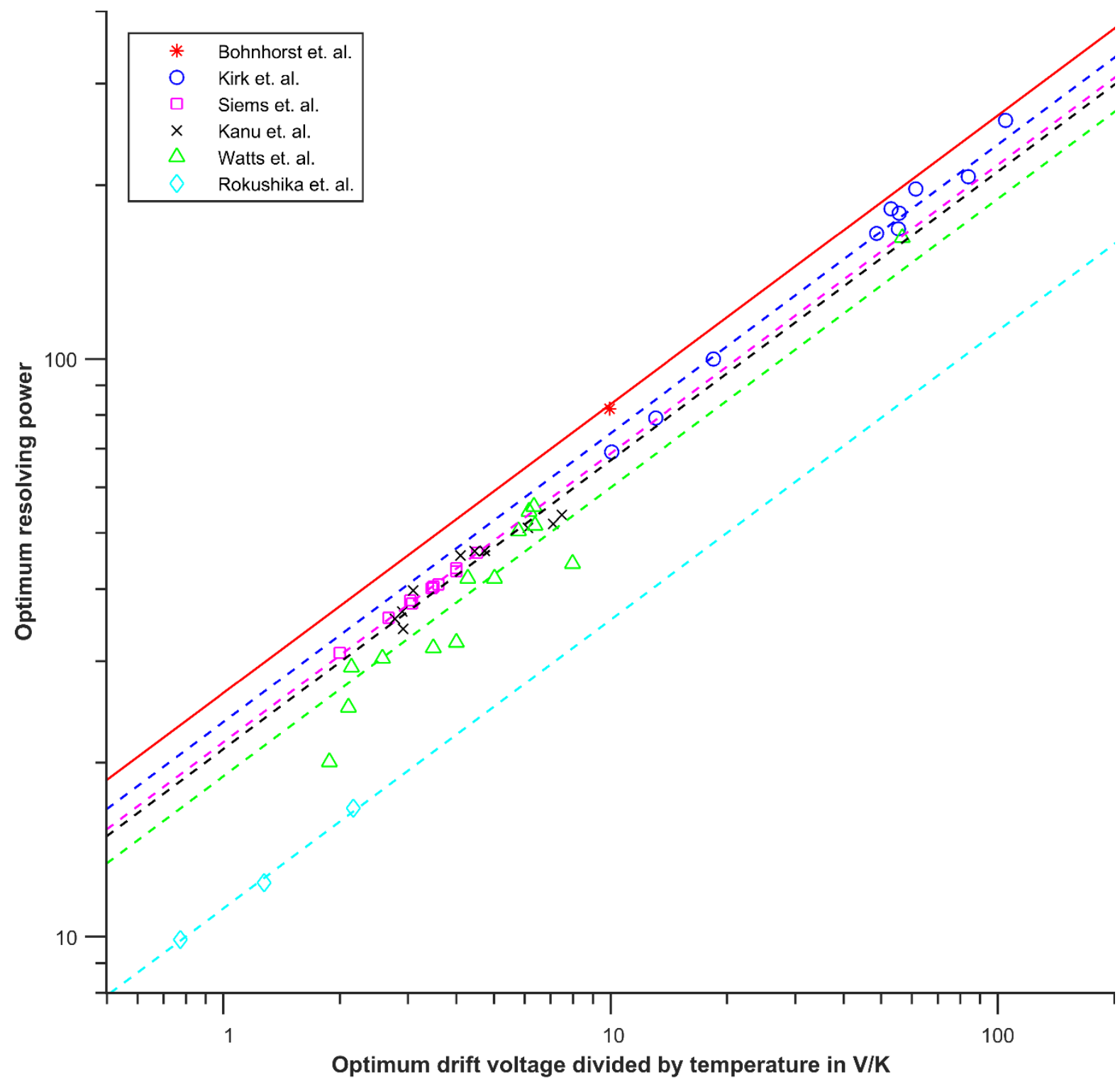

Figure 3: Logarithmic plot of optimum resolving power and voltage from 44 different drift voltage sweeps (symbols), the theoretical curve from eq. 7 (solid red line) and fits to the measurements assuming only an multiplicative error (dashed lines).

Three interesting observations can be made from Figure 3. First, all points lie below the theoretical line from eq. 7 , thus no results contradicting the presented theory were observed. Second, the points from one drift tube design operated under different conditions or build in different length tend to align more or less on the same line, as indicated by the dashed lines in the figure. Thus, the deviation appears to possess a systematic background. This leads to the third point, that this deviation can be interpreted as the "ideality" of the drift tube. The model of eq. 1 and thus also of eq. 7 includes two unavoidable broadening mechanism - diffusion and the minimum width due to injection and amplification. Neither of these can be eliminated. Diffusion must occur during the flight unless it is infinitely short or the temperature is $0 \mathrm{~K}$. No opening time of the shutter would mean that no ions enter the drift tube and an infinitely fast amplifier would create infinite noise. Other broadening mechanisms can however be mitigated by improving the drift tube design without inherently causing disadvantages for the ion separation process. These include:

- Field inhomogeneities inside the drift tube can be prevented through a meticulously designed drift ring arrangement. Both the homogeneity of the created electrical field and the shielding of outside fields have to be considered [10;9]. 
- Field homogeneities at the beginning of the drift tubes can be prevented through a suitable ion shutter design. The use of a field switching shutter provides an advantage over a Bradbury-Nielsen shutter design in this regard [11].

- Additional detector rise times can be avoided through properly shielding the detector with the aperture grid [12; 4].

- Differences between ion mobilities of the same ion species can be minimized by ensuring that all ions experience the same neutral density during their drift. This means creating a homogenous temperature and pressure profile inside the drift tube.

- Inhomogeneities in the drift gas flow profile, which can play a role at extremely high drift gas flows [13], can be avoided by a proper gas inlet and outlet design.

- Chemical broadening of peaks through reactions inside the drift tubes can be minimized by avoiding contamination of both the drift tube material and the drift gas.

- Coulomb repulsion can be mitigated through using a sufficiently small charge density during the drift experiment. While this also reduces signal intensities, coulomb repulsion can still be regarded an avoidable broadening mechanism for two reasons. First, good signal intensities are still achievable at vanishingly small charge densities. Second, more compact drift tube designs are theoretically, at the same limits of detection, significantly less susceptible to coulomb repulsion effects [4].

Most likely, the deviations of the real drift tubes from the ideal curve in Figure 3 can be attributed to various combinations of the above problems.

The ratio between the measured optimum resolving power and the theoretical optimum resolving power for a drift tube possessing its optimum at the measured optimum drift voltage can be calculated according to eq. 8. It can be seen as a measure for how well the drift tube approaches an ideal drift tube only limited by the unavoidable broadening mechanisms diffusion and minimum width. It is especially interesting to note that this measure of ideality also drops when the drift tube exhibits so-called diffusion like broadening effects [2], meaning non-idealities with the same voltage dependence as diffusion.

$$
\text { Ideality }=\frac{1}{26.4} \sqrt{\frac{R_{\text {opt,meas }}{ }^{2} T}{U_{\text {opt }, \text { meas }}}}
$$

The ideality factors for the different designs shown in Figure 3 are summarized in Table 2, which is sorted by the year of publication. A more or less continuous increase in ideality over time can be observed, which may be attributed to a continuous development of drift tube technology. While the earliest designs characterized here only reached less than half the resolving power that should be obtainable at their optimum drift voltage, the current printed circuit board design of Bohnhorst et. al. is able to obtain $99 \%$ of the theoretical value. It is important to note that increased ideality also results in better limits of detection, as a sharper peak of the same charge is also higher.

Table 2: Publication years and ideality factors of different drift tubes

\begin{tabular}{|c|c|c|}
\hline Publication & Year(s) & Ideality \\
\hline Rokushika et. al. [7] & 1985 & $42 \%$ \\
\hline Watts et. al. [8] & 1992 & $72 \%$ \\
\hline Siems et. al. [2] & 1994 & $82 \%$ \\
\hline Kanu et. al. [3] & 2008 & $80 \%$ \\
\hline Kirk et. al. [4; 5] & $2013 / 2015$ & $89 \%$ \\
\hline Bohnhorst et. al. [9] & 2016 & $99 \%$ \\
\hline
\end{tabular}

Another type of drift tube from which one would expect such a performance would be resistive glass, as it should be able to create a perfectly homogenous electrical field. Unfortunately, the literature so far provides only a single paper in which at least partial drift voltage sweeps are shown [14]. Here, determining the correct maximum is rather difficult. However, in agreement with the authors, which mentioned significantly too low resolving powers, an ideality factor of about $50 \%$ can be estimated. This may either, as the authors did, be 
attributed to a non-ideal resistive coating or, equally likely, to the fact that the electric field homogeneity inside the tube is only one of many factors being important for designing an ideal drift tube.

\section{Conclusion}

The analysis shown in this work provides an easy-to-employ tool for judging the quality of a drift tube design. This can be done from a single drift voltage sweep and without any knowledge of further operating parameters apart from the temperature. Thus, this method is as of now the simplest way to both assess new designs or quality control the production of a known drift tube.

\section{Acknowledgements}

Funded by the German Research Foundation (DFG).

\section{References}

1. Revercomb HE, Mason EA (1975) Theory of plasma chromatography/gaseous electrophoresis. Review. Anal. Chem. 47:970-983

2. Siems WF, Wu C, Tarver EE, Hill, Herbert H. Jr., Larsen PR, McMinn DG (1994) Measuring the Resolving Power of Ion Mobility Spectrometers. Anal. Chem. 66:4195-4201

3. Kanu AB, Gribb MM, Hill HH (2008) Predicting optimal resolving power for ambient pressure ion mobility spectrometry. Anal. Chem. 80:6610-6619

4. Kirk AT, Allers M, Cochems P, Langejuergen J, Zimmermann S (2013) A compact high resolution ion mobility spectrometer for fast trace gas analysis. Analyst 138:5200-5207

5. Kirk AT, Zimmermann S (2015) An analytical model for the optimum drift voltage of drift tube ion mobility spectrometers with respect to resolving power and detection limits. Int. J. Ion Mobil. Spec. 18:129-135

6. Kirk AT, Zimmermann S (2015) Pushing a compact $15 \mathrm{~cm}$ long ultra-high resolution drift tube ion mobility spectrometer with $\mathrm{R}=250$ to $\mathrm{R}=425$ using peak deconvolution. Int. J. Ion Mobil. Spec. 18:17-22

7. Rokushika S, Hatano H, Baim MA, Hill HH (1985) Resolution measurement for ion mobility spectrometry. Anal. Chem. 57:1902-1907

8. Watts P, Wilders A (1992) On the resolution obtainable in practical ion mobility systems. International Journal of Mass Spectrometry and Ion Processes 112:179-190

9. Bohnhorst A, Kirk AT, Zimmermann S (2016) Simulation aided design of a low cost ion mobility spectrometer based on printed circuit boards. Int. J. Ion Mobil. Spec. 19:167-174

10. Soppart O, Baumbach JI (2000) Comparison of electric fields within drift tubes for ion mobility spectrometry. Meas. Sci. Technol. 11:1473-1479

11. Kirk AT, Zimmermann S (2014) Bradbury-Nielsen vs. Field switching shutters for high resolution drift tube ion mobility spectrometers. Int. J. Ion Mobil. Spec. 17:131-137

12. Spangler GE (2002) Expanded theory for the resolving power of a linear ion mobility spectrometer. Int. J. Mass Spectrom. 220:399-418

13. Han F, Du Y, Cheng S, Zhou Q, Chen C, KeyongHou, Wang W, Li H (2012) Computational fluid dynamicsMonte Carlo method for calculation of the ion trajectories and applications in ion mobility spectrometry. Int. J. Mass Spectrom. 309:13-21

14. Kwasnik M, Fernandez FM (2010) Theoretical and experimental study of the achievable separation power in resistive-glass atmospheric pressure ion mobility spectrometry. Rapid communications in mass spectrometry:

RCM 24:1911-1918 